This item was submitted to Loughborough's Research Repository by the author.

Items in Figshare are protected by copyright, with all rights reserved, unless otherwise indicated.

\title{
The role of diffusion on the interface thickness in a ventilated filling box
}

PLEASE CITE THE PUBLISHED VERSION

http://journals.cambridge.org/action/displayJournal?jid=FLM

\section{PUBLISHER}

(c) Cambridge University Press

VERSION

VoR (Version of Record)

LICENCE

CC BY-NC-ND 4.0

\section{REPOSITORY RECORD}

Kaye, N.B., M.R. Flynn, Malcolm J. Cook, and Y. Ji. 2019. "The Role of Diffusion on the Interface Thickness in a Ventilated Filling Box". figshare. https://hdl.handle.net/2134/8933. 
This item was submitted to Loughborough's Institutional Repository (https://dspace.lboro.ac.uk/) by the author and is made available under the following Creative Commons Licence conditions.

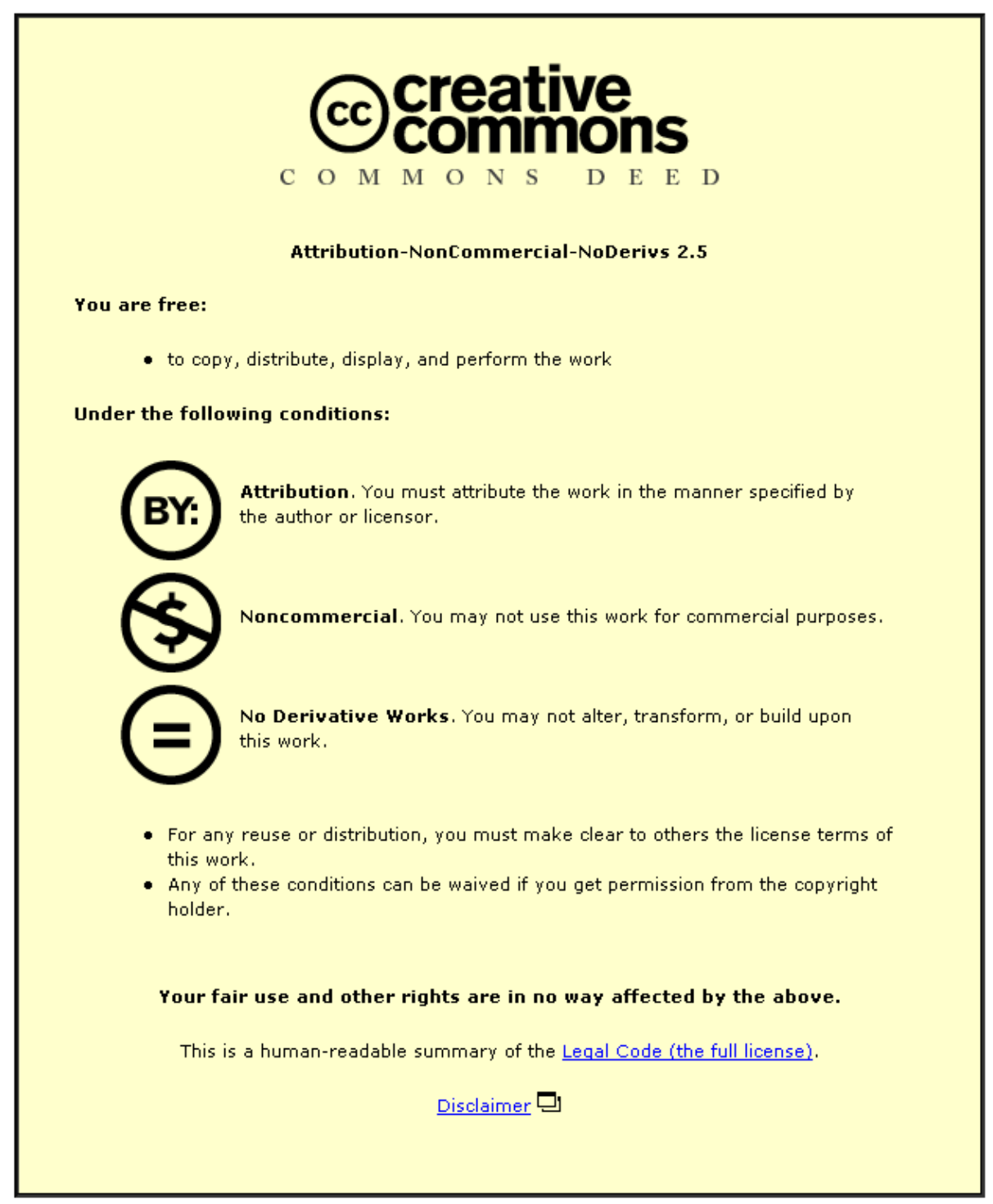

For the full text of this licence, please go to: http://creativecommons.org/licenses/by-nc-nd/2.5/ 


\title{
The role of diffusion on the interface thickness in a ventilated filling box
}

\author{
N. B. $\mathrm{KAYE}^{1}$, M. R. FLYN $\mathrm{N}^{2} \dagger$, M. J. $\operatorname{COOK}^{3}$ AND Y. $\mathrm{JI}^{4}$ \\ ${ }^{1}$ Department of Civil Engineering, Clemson University, Clemson, SC 29634, USA \\ ${ }^{2}$ Department of Mechanical Engineering, University of Alberta, Edmonton, AB, Canada T6G 2G8 \\ ${ }^{3}$ Department of Civil and Building Engineering, Loughborough University, \\ Leicestershire LE11 3TU, UK \\ ${ }^{4}$ Institute of Energy and Sustainable Development, De Montfort University, \\ The Gateway, Leicester LE1 9BH, UK
}

(Received 13 October 2009; revised 10 February 2010; accepted 11 February 2010; first published online 9 April 2010)

We examine the role of diffusivity, whether molecular or turbulent, on the steady-state stratification in a ventilated filling box. The buoyancy-driven displacement ventilation model of Linden et al. (J. Fluid Mech., vol. 212, 1990, p. 309) predicts the formation of a two-layer stratification when a single plume is introduced into an enclosure with vents at the top and bottom. The model assumes that diffusion plays no role in the development of the ambient buoyancy stratification: diffusion is a slow process and the entrainment of ambient fluid into the plume from the diffuse interface will act to thin the interface resulting in a near discontinuity of density between the upper and lower layers. This prediction has been corroborated by small-scale salt bath experiments; however, full-scale measurements in ventilated rooms and complementary numerical simulations suggest an interface that is not sharp but rather smeared out over a finite thickness. For a given plume buoyancy flux, as the cross-sectional area of the enclosure increases the volume of fluid that must be entrained by the plume to maintain a sharp interface also increases. Therefore the balance between the diffusive thickening of the interface and plume-driven thinning favours a thicker interface. Conversely, the interface thickness decreases with increasing source buoyancy flux, although the dependence is relatively weak. Our analysis presents two models for predicting the interface thickness as a function of the enclosure height, base area, composite vent area, plume buoyancy flux and buoyancy diffusivity. Model results are compared with interface thickness measurements based on previously reported data. Positive qualitative and quantitative agreement is observed.

\section{Introduction}

The filling box model, first proposed by Baines \& Turner (1969), provides a prediction for the buoyancy distribution in a sealed enclosure with a single-point source of buoyancy. The model has been applied to mixing in the atmosphere and ocean (Baines \& Turner 1969; Manins 1979), magma chambers (Huppert et al. 1986) and liquid natural gas storage tanks (Germeles 1975). In the filling box analysis, a buoyancy source located in the centre of the enclosure floor creates a plume that rises to the top of the space and forms an expanding buoyant upper layer that is 


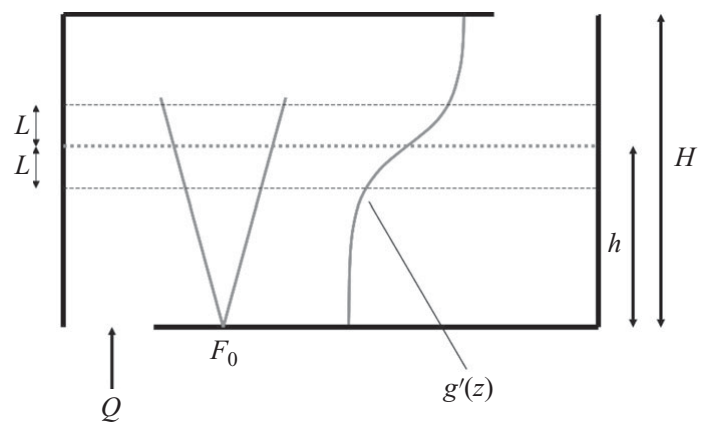

FIGURE 1. A ventilated filling box, of total height $H$, with a nominal interface height $h$ and an interface thickness $2 L$.

separated from the lower ambient layer by a sharp density interface or 'first front'. Baines and Turner's model (Baines \& Turner 1969) was subsequently extended by Linden, Lane-Serff \& Smeed (1990) to consider an enclosure connected by upper and lower vents to an extensive external environment, applicable in describing natural ventilation airflows in buildings. In a ventilated filling box, the presence of the upper buoyant layer creates a pressure difference across the vents, which in turn drives a draining flow. Steady state is realized when this draining flow is balanced by the convective plume flow.

In the models described above, diffusive processes are regarded as slow and molecular transport is therefore ignored. Further, observations of small-scale laboratory experiments show interface thicknesses of less than $1 \mathrm{~mm}$, appearing to confirm the no diffusion assumption. However, just as there is a balance between the draining and filling flows in a ventilated filling box, there is also a balance between the rate at which the interface spreads by diffusion and thins by entrainment into the plume. This paper explores the conditions under which diffusion is important.

Firstly, we consider the time scales relevant to a ventilated filling box flow, as depicted schematically in figure 1 . The transient model for a single enclosure was presented by Kaye \& Hunt (2004). They demonstrated that the time evolution is controlled by the magnitude of the emptying time

$$
T_{e}=\frac{C^{1 / 2} A H^{4 / 3}}{A^{\star} F_{0}^{1 / 3}} \equiv \frac{A}{A^{\star}}\left(\frac{H}{\left.g_{p}^{\prime}\right|_{z=H}}\right)^{1 / 2}
$$

relative to the filling time

$$
T_{f}=\frac{A}{C F_{0}^{1 / 3} H^{2 / 3}} .
$$

Here $T_{e}$ is proportional to the amount of time required for buoyant fluid to drain from a ventilated box after the cessation of convective forcing. Conversely, $T_{f}$ is proportional to the amount of time required for a closed box to fill with buoyant fluid. In the above equations, $A$ is the floor area, $H$ is the enclosure height and $A^{\star}$ is the composite effective vent area defined as

$$
A^{\star}=\frac{2^{1 / 2} A_{T} A_{B}}{\left(A_{T}^{2}+A_{B}^{2}\right)^{1 / 2}}
$$

where $A_{T}$ and $A_{B}$ denote, respectively, the effective vent areas at the ceiling and floor of the enclosure. Furthermore, $C=(6 \alpha / 5)(9 \alpha / 10)^{1 / 3} \pi^{2 / 3}$ is the universal plume 
constant in which $\alpha \simeq 0.1$ is the entrainment coefficient for an axisymmetric, 'top hat' plume. Finally, $F_{0}$ is the plume source buoyancy flux, $z$ is the vertical coordinate measured from the floor and $g_{p}^{\prime}$ is the plume-reduced gravity. For ideal plumes whose source volume flux, $Q_{0}$, is vanishingly small, $\left.g_{p}^{\prime}\right|_{z=H}=C^{-1} F_{0}^{2 / 3} H^{-5 / 3}$ (Baines \& Turner 1969). Because the terminal interface height is controlled by the balance of the filling and emptying processes, it too must be a function of $T_{f}$ and $T_{e}$, or more specifically their ratio

$$
\frac{T_{f}}{T_{e}}=\frac{A^{\star}}{H^{2} C^{3 / 2}} .
$$

Including diffusive effects through a transport coefficient, $\kappa$, adds an additional time scale to the problem, namely the time taken for buoyancy to diffuse over the depth of the enclosure

$$
T_{\kappa}=\frac{H^{2}}{\kappa} .
$$

The interface thickness, $2 L$, is controlled by a balance between diffusion and entrainment and therefore depends on the following ratio of the diffusion and filling time scales:

$$
\frac{T_{\kappa}}{T_{f}}=\frac{C F_{0}^{1 / 3} H^{8 / 3}}{\kappa A} .
$$

Formally, and for reasons that will become clear in $\S 2.2$, it is advantageous to multiply the above ratio by $(2 \alpha)^{4 / 3} C^{-1}$ and thereby define a non-dimensional parameter $\mathscr{R}$ such that

$$
\mathscr{R} \equiv \frac{(2 \alpha)^{4 / 3}}{C} \cdot \frac{T_{\kappa}}{T_{f}}=\frac{(2 \alpha)^{4 / 3} F_{0}^{1 / 3} H^{8 / 3}}{\kappa A},
$$

where $\mathscr{R}$ is a ratio relating the rate of convection to diffusion, similar to the Péclet number $P e$, which is a ratio relating the rate of advection to diffusion.

For $\mathscr{R} \gg 1$, diffusion plays only a minor role and a relatively thin interface is anticipated. This is typically the case for similitude experiments of full-scale ventilation flows. In a typical experiment, $H \simeq 30 \mathrm{~cm}, A \simeq 30 \mathrm{~cm} \times 20 \mathrm{~cm}$ and $F_{0} \simeq 250 \mathrm{~cm}^{4} \mathrm{~s}^{-3}$ (see e.g. Lin \& Linden 2002). Selecting the molecular value for the diffusion coefficient appropriate for salt in water yields $\mathscr{R} \simeq 7.5 \times 10^{5}$. A smaller, possibly significantly smaller, value for $\mathscr{R}$ would be obtained if one chose an eddy, rather than the molecular, diffusivity. However, the magnitude of the eddy diffusivity depends upon the flow and entrainment characteristics inside the particular experimental apparatus; detailed information of this type is typically not recorded or reported in similitude experimental modelling. Conversely, in a full-scale ventilation flow, we consider $F_{0} \simeq$ $5.4 \times 10^{-3} \mathrm{~m}^{4} \mathrm{~s}^{-3}$, corresponding to a $10^{\circ} \mathrm{C}$ temperature difference between the source and the surroundings and a source flow rate of 1 air change per hour for a room of dimensions $3 \mathrm{~m}$ tall by $20 \mathrm{~m}^{2}$ plan area. Selecting again a value for $\kappa$ based on molecular diffusion, in this case for heat through air, gives $\mathscr{R} \simeq 9.2 \times 10^{2}$, roughly $10^{3}$ times less than the previous estimate. (Consistent with the above remarks, a much smaller value of $\mathscr{R}$ would apply to chambers with significant background velocity fluctuations.) One might therefore expect notably more diffuse interfaces at full scale rather than reduced scale (Howell \& Potts 2002). To elucidate these details, a major goal of this paper is to estimate, using a pair of complementary analytical approaches, the functional relationship between the interface thickness, the chamber geometry and ratio of time scales given by (1.5). That is, we seek to establish the nature of the function $L / H=L / H\left(A^{\star} / H^{2}, \mathscr{R}\right)$. 
In $\S 2$, we present a first-order phenomenological model that balances the rate of thickening of the interface due to diffusion with the rate of thinning due to entrainment into the plume. Also discussed are the integral plume equations that describe a ventilated filling box. Results are contrasted against measured CFD and experimental data as reported in earlier investigations; this discussion appears in $\S 3$. Finally, in $\S 4$, a series of conclusions are drawn.

\section{Role of diffusion}

\subsection{Approximate solution}

We begin with the standard solution to the one-dimensional diffusion equation for an initial step change in the concentration $\Upsilon$ of an active scalar, say heat or salt:

$$
\Upsilon(z, t)=\frac{\Upsilon_{0}}{2}\left(1+\operatorname{erf}\left[\frac{z}{(4 \kappa t)^{1 / 2}}\right]\right) .
$$

At arbitrary time $t$, the interface can be regarded as having diffused a distance

$$
L \sim(4 \kappa t)^{1 / 2} .
$$

Alternatively, we can regard the diffusion front as a front moving with velocity

$$
u=\frac{\mathrm{d} L}{\mathrm{~d} t}=\frac{2 \kappa}{L}
$$

relative to the stationary terminal interface height.

Now consider a ventilated enclosure with an initially sharp interface at height $h$. Over time the (two-sided) interface will grow in volume with a time rate of change given by

$$
(\Delta Q)_{\text {diffusion }}=2 A \frac{\mathrm{d} L}{\mathrm{~d} t}=\frac{4 \kappa A}{L} .
$$

In addition, buoyant fluid enters the bottom of the diffuse layer and is extracted from the top via the plume. Ignoring stratification effects (This omission is justified a-posteriori by the results of figure 3 ), and assuming that the rising flow remains in pure plume balance, the plume volume flux is given by $Q=C F_{0}^{1 / 3}\left(z+z_{0}\right)^{5 / 3}$ (Woods, Caulfield \& Phillips 2003). Here $z_{0}=\left[Q_{0} /\left(C F_{0}^{1 / 3}\right)\right]^{3 / 5}$ is the distance of the virtual source below the actual source. The net rate at which fluid is extracted from the diffuse interface is therefore

$$
(\Delta Q)_{\text {entrainment }}=C F_{0}^{1 / 3}\left[\left(h+z_{0}+L\right)^{5 / 3}-\left(h+z_{0}-L\right)^{5 / 3}\right] .
$$

Balancing the rates of diffusion and entrainment yields

$$
\begin{aligned}
\frac{4 \kappa A}{L} & =C F_{0}^{1 / 3}\left[\left(h+z_{0}+L\right)^{5 / 3}-\left(h+z_{0}-L\right)^{5 / 3}\right] \Leftrightarrow \\
\frac{1}{\mathscr{R}} & =\frac{3}{20}\left(\frac{9}{20}\right)^{1 / 3} \pi^{2 / 3} \frac{L}{H}\left[\left(\zeta_{s s}+\zeta_{0}+\frac{L}{H}\right)^{5 / 3}-\left(\zeta_{s s}+\zeta_{0}-\frac{L}{H}\right)^{5 / 3}\right],
\end{aligned}
$$

where $\zeta_{s s}=h / H$ and $\zeta_{0}=z_{0} / H$. Physical insights into the solution structure can be ascertained by expanding the square-bracketed terms from (2.6) as Taylor series. When the interface is relatively thin and not close to the lower boundary so that 
(a)

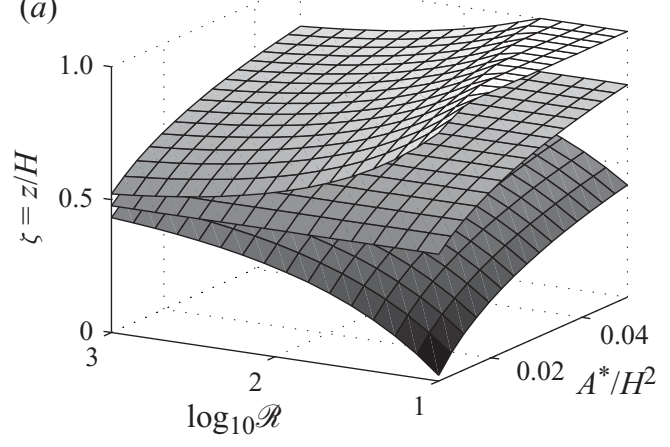

(b)

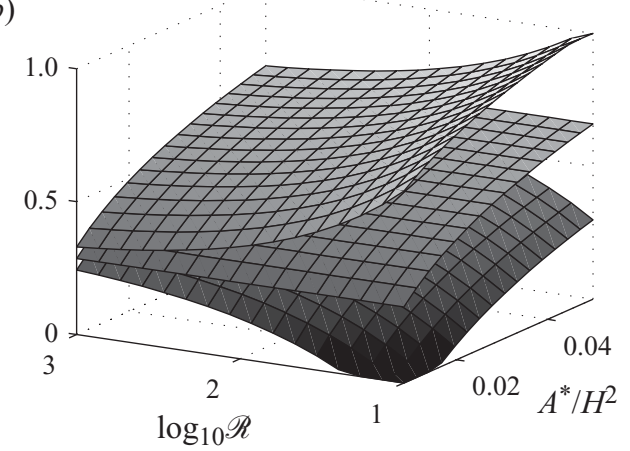

FiguRE 2. (a) Ideal plume: normalized interface height as a function of $A^{*} / H^{2}$ (middle surface) as determined from (2.9). Surfaces showing the thickness of the diffuse interface are also presented; these are based upon the solution to (2.6) with $\zeta_{0}=0$. (b) Non-ideal plume: as in $(a)$ but with $\zeta_{0}=0.25$. The interface height is now determined by solving (2.10).

$L / H \ll \zeta_{s s}+\zeta_{0}$, the leading-order solution is given by

$$
L \simeq\left[\frac{6 \kappa A}{5 C F_{0}^{1 / 3}\left(h+z_{0}\right)^{2 / 3}}\right]^{1 / 2} \Leftrightarrow \frac{L}{H} \simeq\left(\frac{2}{\mathscr{R}}\right)^{1 / 2}\left[\frac{2 \times 5^{1 / 2}}{3 \pi\left(\zeta_{s s}+\zeta_{0}\right)}\right]^{1 / 3} .
$$

In the opposite limit, i.e. $L / H \gg \zeta_{s s}+\zeta_{0}$,

$$
L \simeq\left(\frac{2 \kappa A}{C F_{0}^{1 / 3}}\right)^{3 / 8} \Leftrightarrow \frac{L}{H} \simeq\left(\frac{20}{9 \pi^{2}}\right)^{1 / 8}\left(\frac{10}{3 \mathscr{R}}\right)^{3 / 8} .
$$

Here the interface is both thick and close to the floor; the small parameters $\zeta_{s s}$ and $\zeta_{0}$ do not appear in (2.8). Consistent with Baines (1983), (2.7) and (2.8) indicate that the interface thickness, $2 L$, grows with increasing floor area and diffusion coefficient. By contrast, $2 L$ will be relatively small for larger plume buoyancy fluxes.

The connection between (2.6) and the chamber geometry and source conditions is elucidated as follows: when the plume is ideal i.e. $Q_{0}=0, \zeta_{s s}$, can be determined from

$$
\frac{A^{\star}}{H^{2}}=C^{3 / 2}\left(\frac{\zeta_{s s}^{5}}{1-\zeta_{s s}}\right)^{1 / 2}
$$

(Linden et al. 1990). When the source is non-ideal and $A_{T}=A_{B}, \zeta_{s s}$ is determined instead from (Woods et al. 2003)

$$
\frac{A^{\star}}{H^{2}}=\frac{C^{3 / 2}\left(\zeta_{s s}+\zeta_{0}\right)^{5 / 6}}{\left(1-\zeta_{s s}\right)^{1 / 2}}\left[\left(\zeta_{s s}+\zeta_{0}\right)^{10 / 3}+\frac{1}{2} \zeta_{0}^{10 / 3}-\zeta_{0}^{5 / 3}\left(\zeta_{s s}+\zeta_{0}\right)^{5 / 3}\right]^{1 / 2}
$$

In figure 2, we consider solutions to (2.6), (2.9) and (2.10) for a range of $A^{*} / H^{2}$ and $\mathscr{R}$ and a pair of values for $\zeta_{0}$.

\subsection{Ventilated filling box model}

So as to rigorously verify the above predictions, we couple the integral plume equations and the ambient density equation. It is assumed that the system is Boussinesq and that the plume is adequately described by a 'top-hat' representation. The former equations quantify the vertical variation of the plume volume flux $\pi Q$, momentum flux, $\pi M$ and 
buoyancy flux, $\pi F$; the latter equation specifies the spatial-temporal variation of the reduced gravity $g^{\prime} \equiv g\left(\rho_{a}-\bar{\rho}_{p}\right) / \rho_{00}$. Here $\rho_{00}$ is a reference density, $\rho_{a}$ is the ambient density and $\bar{\rho}_{p}$ is the horizontal-average plume density. Using subscripts to indicate differentiation with respect to the vertical coordinate $z$ and time $t$, the equations read (see e.g. Germeles 1975; Manins 1979)

$$
\begin{gathered}
Q_{z}=2 \alpha M^{1 / 2}, \quad M_{z}=\frac{Q F}{M}, \\
F_{z}=-Q g_{z}^{\prime}, \quad g_{t}^{\prime}+\frac{Q_{v}-\pi Q}{A} g_{z}^{\prime}=\kappa g_{z z}^{\prime} .
\end{gathered}
$$

In the above, the advection velocity of $(2.12 b)$ is equal to the background ambient velocity, which is itself proportional to the difference between the ventilation flow rate $Q_{v}$ and the local plume volume flux $\pi Q$.

With the notable exception of Manins (1979), many previous investigations follow the assumptions applied by Baines \& Turner (1969) whereby diffusion is omitted and $g_{t}^{\prime}$ tends to a constant in the long-time limit. Herein, we return to Manins's approach, albeit in a ventilated rather than a closed filling box, and instead consider steady solutions with a non-vanishing diffusion coefficient. Non-dimensional variables are introduced such that

$$
\begin{gathered}
g^{\prime}=\frac{F_{0}^{2 / 3} \delta}{(2 \alpha)^{4 / 3} H^{5 / 3}}, \quad z=H \zeta, \quad F=F_{0} f, \\
Q=(2 \alpha)^{4 / 3} F_{0}^{1 / 3} H^{5 / 3} q, \quad M=(2 \alpha)^{2 / 3} F_{0}^{2 / 3} H^{4 / 3} m .
\end{gathered}
$$

Applying (2.13) and (2.14) in (2.11) and (2.12) with $g_{t}^{\prime} \rightarrow 0$ yields

$$
\begin{gathered}
q_{\zeta}=m^{1 / 2}, \quad m_{\zeta}=\frac{q f}{m}, \\
f_{\zeta}=-q \delta_{\zeta}, \quad \gamma_{\zeta}=\mathscr{R} \gamma\left(q_{v}-\pi q\right), \quad \delta_{\zeta}=\gamma,
\end{gathered}
$$

where $\mathscr{R}$, whose magnitude dictates the stiffness of the above system of ordinary differential equations, is defined by (1.6) and the second-order equation (2.12b) has been broken into the two first-order equations $(2.16 b, c)$.

Boundary conditions appropriate to the case of an ideal plume are $q(0)=0$, $m(0)=0, f(0)=1$ and $\delta(0)=0$. The numerical value of $\gamma(0)$ is selected such that $f(1)=0$, i.e. the plume buoyancy flux at the top of the chamber vanishes. Unless the interface naturally lies close to the upper boundary and/or $\mathscr{R} \lesssim \mathcal{O}\left(10^{1}\right)$ such that the interface is especially broad, $f(1)=0 \Rightarrow \delta(1)=\pi / q_{v} \Leftrightarrow g^{\prime}(H)=\pi F / Q_{v}$, the latter condition being consistent with the analysis of Linden et al. (1990). When solving the system of ordinary differential equations, a pair of iterations are necessary. For prescribed $q_{v}$ and from a pair of sensible initial guesses for $\gamma(0)(>0)$, we converge to that unique value of $\gamma(0)$ satisfying $f(1)=0$. Then, by integrating $\delta$ with height, the actual non-dimensional ventilation flow rate may be evaluated from (cf. (2.15) of Linden et al. 1990)

$$
q_{v}=\frac{1}{4 \alpha^{2}}\left(\frac{A^{\star}}{H^{2}}\right)\left(\int_{0}^{1} \delta \mathrm{d} \zeta\right)^{1 / 2} \quad \Leftrightarrow \quad\left(\frac{Q_{v}}{A^{\star}}\right)^{2}=\int_{0}^{H} g^{\prime} \mathrm{d} z .
$$

The original estimate for $q_{v}$ is updated accordingly and we repeat the above steps until a tolerance of $1 \times 10^{-7}$ on $q_{v}$ is achieved. With the initial conditions given previously, 


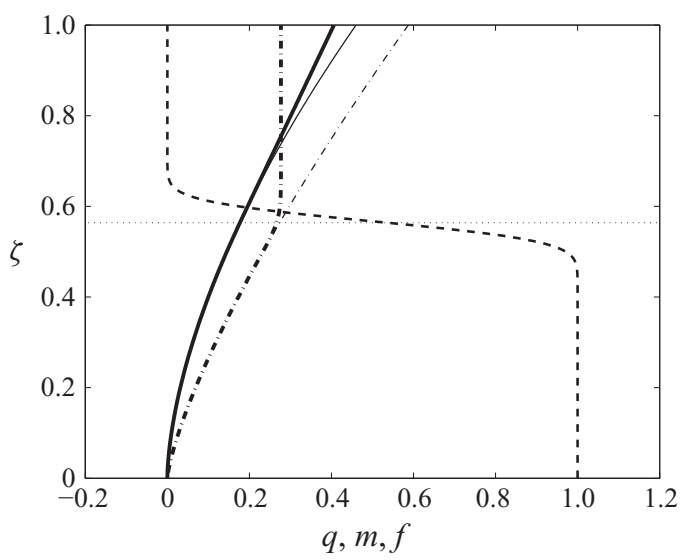

FIGURE 3. Solutions to the (diffusive) filling box equations (2.15)-(2.16) with $A^{\star} / H^{2}=1.41 \times 10^{-2}, \mathscr{R}=500$ and $\zeta_{0}=0$. The dashed and thick solid and dash-dotted lines show, respectively, $f, q$ and $m$. The thin solid and dash-dotted lines are described in the text and the thin horizontal line shows the vertical location, $\zeta=0.564$, where $q=q_{v} / \pi$.

solution behaviour is then governed by the pair of non-dimensional parameters $\mathscr{R}$ and $A^{\star} / H^{2}$. Whereas a formal investigation of the convergence characteristics for the above algorithm is deferred to a future investigation, we note in passing that the present approach is largely successful provided $\mathscr{R} \lesssim 10^{3}-10^{4}$ (i.e. the system stiffness is allowed to be large but not extreme) and the centre of the interface (i.e. the location where $\pi q=q_{v}$ and, from (2.16b) and (2.16c), where $\delta_{\zeta}$ attains its maximum value) is not too close to the upper boundary.

Representative output for a particular combination of $A^{\star} / H^{2}$ and $\mathscr{R}$ is given in figure 3. It shows vertical profiles of $q, m$ and $f$ as determined from $(2.15 a),(2.15 b)$ and $(2.16 a)$, respectively. Outside of the interfacial region, the buoyancy flux is nearly constant, either $f \simeq 1$ in the lower layer or $f \simeq 0$ in the upper layer. Because $f$ becomes vanishingly small as $\zeta$ tends to $1, m$ (denoted by the thick dash-dotted line) approaches a constant value and $q$ (denoted by the thick solid line) becomes a linear function of height. For reference, the thin solid and dash-dotted lines of figure 3 show, respectively, vertical profiles of $q$ and $m$ for the special case of an unstratified ambient with $f(\zeta)=1$. The deviation between the thick and thin dash-dotted lines begins abruptly near the vertical location where $\pi q$ and $q_{v}$ coincide. Conversely, the thick and thin solid lines do not deviate significantly from one another except relatively close to the top of the enclosure, i.e. $\zeta \gtrsim 0.7$. Note that exact solutions may be derived when $f(\zeta)=1$ (Morton, Taylor \& Turner 1956) and have the form $q=(3 / 5)(9 / 20)^{1 / 3} \zeta^{5 / 3}, m=(9 / 20)^{2 / 3} \zeta^{4 / 3}$.

Further solutions to the (diffusive) filling box equations are presented in figure 4 , which considers two different values of $A^{\star} / H^{2}$, and $\mathscr{R}$ spanning two orders of magnitude. For clarity, vertical profiles of $q$ and $m$ are omitted, however, the nondimensional vertical density distribution is now indicated (solid curve). Whereas the centre of the interface changes relatively little with $\mathscr{R}$, the interface thickness is observed to decrease sharply with increasing $\mathscr{R}$ in both figure $4(a-d)$ and figure $4(e-h)$. This trend is quantitatively well captured by (2.6) whose predictions are demarcated by the upper and lower dotted horizontal lines and which provides a reliable prediction for the interfacial thickness over a broad range of $\mathscr{R}$. 

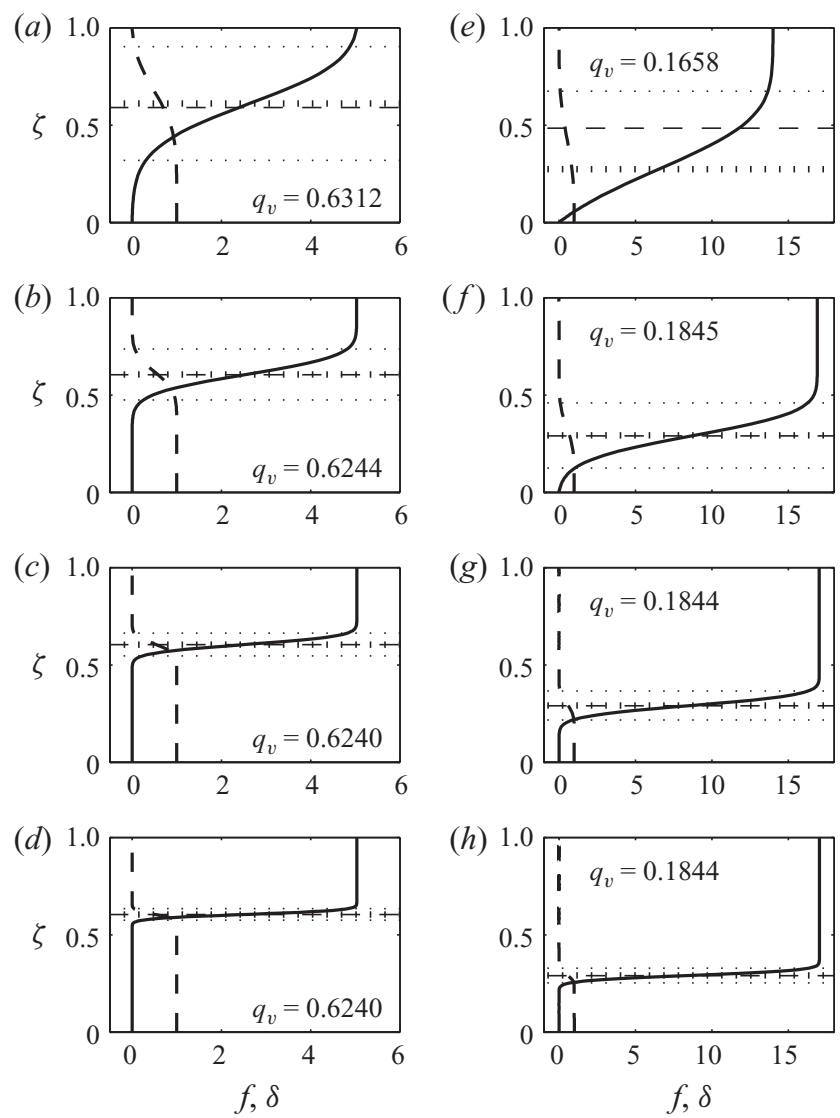

FIGURE 4. Vertical distributions of $f$ (dashed line) and $\delta$ (solid line) for $\zeta_{0}=0$, $A^{\star} / H^{2}=1.77 \times 10^{-2}(a-d), A^{\star} / H^{2}=2.12 \times 10^{-3}(e-h)$ and $(a, e) \mathscr{R}=20,(b, f) \mathscr{R}=100$, $(c, g) \mathscr{R}=500$ and $(d, h) \mathscr{R}=2 \times 10^{3}$. The middle dotted horizontal line (thick) shows the point at which $q=q_{v} / \pi$ and the upper and lower dotted horizontal lines (thin) indicate the interfacial thickness based on the estimate of (2.6). The dashed horizontal line shows the equivalent interface height assuming an upper layer of uniform buoyancy with $\delta=\pi / q_{v}$.

\section{Comparison with previous analyses}

For most cases of architectural interest, and consistent with figure 4, (2.7) is the more relevant approximation to (2.6) than (2.8). Here, we contrast the thin interface approximation (2.7) with previously published measurements of buoyancy profiles in model ventilated enclosures. A representative set of scaled temperature profiles from Kaye, Ji \& Cook (2009) is shown in figure 5 along with the interface height predicted from Linden et al. (1990) and the upper and lower bounds on the interface thickness predicted from (2.7). Positive agreement between the simple scaling model and the full simulation results is observed suggesting that diffusion is the primary cause of interfacial thickening.

Estimates of the interface thickness for the salt bath experiments of Kaye \& Hunt (2004) predict an interface thickness in the range of $0.5-0.75 \mathrm{~mm}$. While accurate measurement of this thickness was not possible during those experiments, laboratory images show the steady-state interface to be extremely sharp as can be observed, most especially, from figure 13 of Kaye and Hunt's paper. 

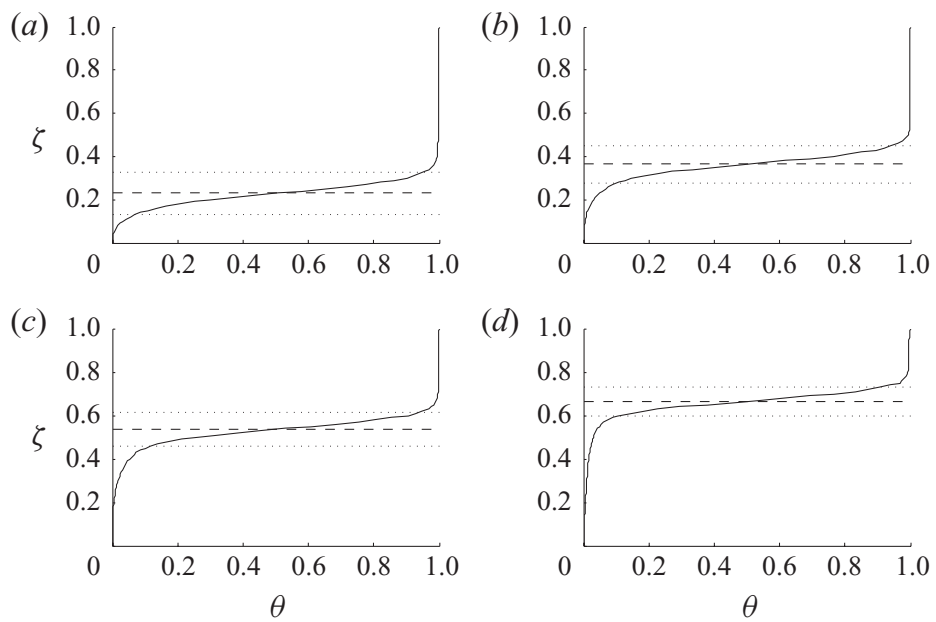

FIGURE 5. Scaled temperature profiles, $\theta=\left(T-T_{\text {floor }}\right) /\left(T_{\text {ceiling }}-T_{\text {floor }}\right)$, plotted against $\zeta$, based on the CFD simulations of Kaye et al. (2009), with the interface limits predicted by (2.7) indicated by the horizontal dotted lines. The horizontal dashed lines show the interface height predicted by Linden et al. (1990). (a) $A^{\star} / H^{2}=0.0026$, (b) $A^{\star} / H^{2}=0.0078$ (c) $A^{\star} / H^{2}=0.0208$ and $(d) A^{\star} / H^{2}=0.0624$.

Quantitative comparison with full scale results is problematic due to a lack of published data regarding the detailed vertical temperature profile in a full-scale room with well-categorized heat inputs. One might argue that the near full-scale data of Howell \& Potts (2002) could suffice. However, in Howell \& Potts's experiments, calculations based on their published data and standard heat transfer correlations show that their heat source had an operating temperature of approximately $165^{\circ} \mathrm{C}$. This is significantly hotter than typical surfaces in most commercial buildings indicating that the collected data may be unduly influenced by radiative and nonBoussinesq effects. As such, there are different, and more complicated, heat transfer effects that come into play in interpreting Howell and Potts' measured results (Howell \& Potts 2002), making a straightforward comparison to the present models nontrivial. Even so, it is worth pointing out the qualitative similarities between the vertical buoyancy profiles of figures 3, 5, 8 and 9 from their paper and the solid curves of figure $4(e, f)$. Whereas Howell \& Potts (2002) suggest that radiative effects must be incorporated in order to generate buoyancy profiles of such a distinctive shape (i.e. nearly linearly stratified through the lower layer with a uniform temperature in the upper layer), our analysis demonstrates that diffusive effects alone suffice. In a similar vein, Howell and Potts' criticisms of the two-layer model of Linden et al. (1990) as being practically unsound are, in our opinion, overzealous. Howell $\&$ Potts (2002) argue that application of equations by Linden et al. (1990) may lead to qualitatively erroneous predictions, for example, with respect to the vertical stratification of temperature. Unlike (2.6), (2.7) or (2.8) however, their discussion considers a single source buoyancy flux and chamber geometry. Moreover, as we indicate quantitatively in figure 4 , the ventilation flow rate, another key parameter in ventilation design, changes little with $\mathscr{R}: q_{v}$ depends on the total amount of buoyancy within an enclosure rather than its vertical distribution. 


\section{Discussion and conclusions}

Filling box models such as that first proposed by Baines \& Turner (1969) have been broadly applied in describing isolated convection in closed and ventilated geometries. Consistent with their analogue salt-bath experiments, Baines and Turner's equations neglect diffusion so that there is a discontinuous density jump across the 'first front', the interface that separates the uncontaminated ambient from the fluid that previously originated from, or was entrained into, the plume. While this modelling approach can accurately predict, among other quantities, the ventilation flow rate (Linden et al. 1990), it may fail to reproduce the distributed vertical temperature profiles that are often noted, for example, in full-scale measurements of naturally ventilated buildings (Howell \& Potts 2002).

From phenomenological arguments, we give via (2.6) and its approximates equations for the interface thickness as a function of the diffusion and entrainment coefficients, respectively, $\kappa$ and $\alpha$; the plan area of the enclosure $A$; the chamber height $H$; the source volume and buoyancy fluxes; and the area of the openings that connect the enclosure to a much more voluminous ambient. Model predictions are corroborated by solving the filling box equations, (2.15) and (2.16), where, as with the earlier analysis of Manins (1979), $\kappa$ is assumed to be non-zero. In both cases, the interface thickness is predicted to be a decreasing function of $\mathscr{R}$, defined by (1.6), which is analogous to the Péclet number, $P e$. Comparisons are also drawn against related measurements from previous CFD, experimental and full-scale analyses as summarized in $\S 3$. Generally positive agreement is noted.

Viewed from a different perspective, (2.6) or the (diffusive) filling box equations may be applied in estimating a representative ambient diffusion coefficient $\kappa$ in architectural or environmental instances where source conditions and a vertical gradient of temperature are well known. Such an approach has the benefit of simplicity since detailed microstructure measurements are not required, albeit at the expense of providing average rather than spatially detailed predictions for the eddy diffusion coefficient.

Financial support was generously provided by Clemson University and NSERC through the Discovery Grant programme. The helpful comments of three anonymous referees are acknowledged with thanks.

\section{REFERENCES}

Baines, W. D. 1983 Direct measurement of volume flux of a plume. J. Fluid Mech. 132, 247-256.

Baines, W. D. \& TuRner, J. S. 1969 Turbulent buoyant convection from a source in a confined region. J. Fluid Mech. 37, 51-80.

Germeles, A. E. 1975 Forced plumes and mixing of liquids in tanks. J. Fluid Mech. 71, 601-623.

Howell, S. A. \& PotTs, I. 2002 On the natural displacement flow through a full-scale enclosure and the importance of the radiative participation of the water vapour content of the ambient air. Build. Environ. 37, 817-823.

Huppert, H. E., Sparks, R. S. J., Whitehead, J. A. \& Hallworth, M. A. 1986 Replenishment of magma chambers by light inputs. J. Geophys. Res. 91, 6113-6122.

Kaye, N. B. \& Hunt, G. R. 2004 Time-dependent flows in an emptying filling box. J. Fluid Mech. 520, $135-156$.

KAYE, N. B., Ji, Y. \& CoOK, M. J. 2009 Numerical simulation of transient flow development in a naturally ventilated room. Build. Environ. 44, 889-897.

LiN, Y. J. P. \& Linden, P. F. 2002 Buoyancy-driven ventilation between two chambers. J. Fluid Mech. 463, 293-312. 
Linden, P. F., Lane-Serff, G. F. \& Smeed, D. A. 1990 Emptying filling boxes: the fluid mechanics of natural ventilation. J. Fluid Mech. 212, 309-335.

Manins, P. C. 1979 Turbulent buoyant convection from a source in a confined region. J. Fluid Mech. 91, 765-781.

Morton, B. R., Taylor, G. I. \& Turner, J. S. 1956 Turbulent gravitational convection from maintained and instantaneous sources. Proc. R. Soc. A 234, 1-23.

Woods, A. W., Caulfield, C. P. \& Phillips, J. C. 2003 Blocked natural ventilation: the effect of a source mass flux. J. Fluid Mech. 495, 119-133. 